\title{
Enfermedad celíaca del adulto: Experiencia clínica
}

\author{
Carla Mancilla $A^{1}$, Ana María Madrid S1 , Jorge Valenzuela $E^{1,2}$, \\ Antonio Morales $B^{1}$, Carmen H urtado $\mathrm{H}^{1 \mathrm{a}}$, G ladys Smok $\mathrm{S}^{3}$, \\ Rodrigo Ledezma $R^{1 b}$, Iván Castillo $M^{1 b}$, Mauricio Rivas $Q^{1 c}$, \\ Javier Brahm B ${ }^{1,2}$.

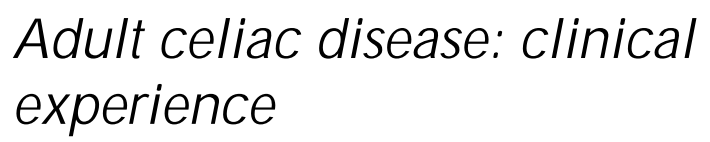

Background: The prevalence of celiac disease (CD) is unknown in Chile. We have recently noted a rise in the number of cases diagnosed among adults. Aim: To describe the clinical characteristics of a group of adult celiac patients. Patients and methods: Clinical data of patients older than 15 years with positive antitransglutaminase or antiendomysial autoantibodies and a duodenal biopsy characteristic of CD were retrospectively reviewed. Age at diagnosis, symptoms and signs and laboratory, endoscopic and histological findings, were analyzed. Results: Thirty seven patients ( 28 women), were studied. Median age at diagnosis was 41 years (range 15-69). Main symptoms and signs were diarrhea (78\%), weight loss (38\%) and abdominal pain (38\%). Anemia was found in $49 \%$, elevation of ESR in $57 \%$, elevation of alkaline phosphatases in $54 \%$, elevation of aspartate aminotransferase in $38 \%$ and a rise in alanine aminotransferase in $27 \%$. Antiendomysial antibodies were positive in $17 / 22$ (77\%) and antitransglutaminase in 19/22 (86\%) patients. Endoscopic findings were suggestive of CD in $47 \%$ of cases and duodenal biopsy showed intestinal villi atrophy in 34 (92\%) patients. The three patients with normal histology had positive serology and a good response to gluten free diet. Conclusions: CD should be considered in the differential diagnosis of patients with unespecific digestive symptons, even when they present late in adult life. Serologic markers are a good diagnostic tool. A normal duodenal pathology does not exclude the diagnosis, if other diagnostic features are present (Rev Méd Chile 2005; 133: 1317-21).

(Key Words: Celiac disease; Gluten; Transglutaminase)

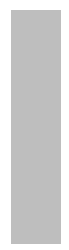

\footnotetext{
Recibido el 4 de diciembre, 2004. Aceptado el 13 de junio, 2005.

${ }^{1}$ Sección de Gastroenterología, Departamento de Medicina, Hospital Clínico de la Universidad de Chile. ${ }^{2}$ Departamento de Gastroenterología, Clínica Las Condes. ${ }^{3}$ Departamento de Anatomía Patológica, Hospital Clínico Universidad de Chile. Santiago, Chile. aQuímico Farmacéutico

bInterno de Medicina, Facultad de Medicina, Universidad de Chile

cAlumno de Medicina, Facultad de Medicina, Universidad de Chile
}

Correspondencia a: Dr. Javier Brahm B. Sección de Gastroenterología, Departamento de Medicina. Hospital Clínico Universidad de Chile. Santos Dumont 999. Fono: 9788350. Fax: 9788349. E mail: jbrahm@redclinicauchile.cl 
La a prevalencia de la enfermedad celíaca (EC) en Chile no se conoce con exactitud, pero aparentemente se está reconociendo mejor en el último tiempo. Habitualmente se diagnostica en la infancia en presencia de diarrea crónica, malnutrición, detención en el desarrollo y atrofia de las vellosidades en biopsias duodenales ${ }^{1,2}$. No obstante, se ha señalado en los últimos años que el diagnóstico puede hacerse con la detección de anticuerpos en sangre, especialmente los antiendomisio (AE) y antitransglutaminasa (ATG). Esto ha permitido hacer el diagnóstico con técnicas menos invasivas y con más frecuencia en adultos, en quienes el cuadro clínico es menos específico.

De acuerdo con esta tendencia, hemos observado un notorio aumento en el diagnóstico de EC en adultos estudiados recientemente, que a menudo se presentan con síntomas y signos que no son los tradicionalmente descritos.

El objetivo de esta comunicación es analizar las características clínicas y de laboratorio de un grupo de pacientes mayores de 15 años en quienes diagnosticamos EC durante los últimos tres años.

\section{PACIENTES Y MÉTODO}

El siguiente es un estudio retrospectivo en que se revisaron los antecedentes clínicos, de laboratorio, endoscópicos e histológicos de 37 pacientes mayores de 15 años de edad con diagnóstico de EC atendidos y diagnosticados "de novo" durante los años 2001 a 2003 en el Hospital Clínico de la Universidad de Chile y en la Clínica Las Condes. La pesquisa de los pacientes se realizó mediante la revisión de las biopsias duodenales y de los registros de pacientes que se realizaron anticuerpos AE y ATG en los respectivos laboratorios. El diagnóstico se fundamentó en los hallazgos histológicos característicos de $\mathrm{EC}$ en la biopsia duodenal obtenida de la segunda porción del duodeno durante una endoscopia digestiva alta o por la presencia de serología positiva AE, ATG o ambos. Los anticuerpos AE IgG e IgA se determinaron mediante técnica de inmunofluorescencia indirecta con sustrato de esófago de mono (Immuno Glo Anti-Endomysial Antibody, IMMCO Diagnostics), considerando el resultado como positivo con lectura $\geq 1: 2,5$. Los anticuerpos ATG IgA se deter- minaron por reacción de ELISA con sustrato de transglutaminasa tisular humana (Quanta Lite htTG IgA INOVA), considerando positivo un valor sobre $20 \mathrm{U} / \mathrm{ml}$.

Para cada paciente se registraron en una ficha tipo datos clínicos como edad al diagnóstico, síntomas y signos de presentación, índice de masa corporal (IMC) y los hallazgos de laboratorio del hemograma, perfil bioquímico y perfil hepático.

Se registraron, además, los hallazgos endoscópicos e histológicos. Estos últimos se clasificaron según la clasificación de Marsh: enteritis linfocítica (I), enteritis linfocítica con hiperplasia críptica (II), atrofia parcial (III A), atrofia subtotal (III B) y atrofia total de las vellosidades o mucosa plana (III C) ${ }^{3}$.

Como se trata de un estudio descriptivo, se presentan los datos en términos de frecuencia y porcentajes.

\section{Resultados}

De los 37 pacientes, 28 (76\%) eran mujeres y 9 (24\%) hombres. Todos cumplían los criterios señalados para hacer el diagnóstico de EC. La edad promedio al momento del diagnóstico fue de 41 \pm 13 años (15-69). El IMC promedio fue de $20,7 \pm 3,7$ (14,3-30). Once (29\%) de los 37 pacientes presentaban IMC $<20$. Las manifestaciones clínicas más importantes están señaladas en la Tabla 1. Los síntomas suman más de 37 por tener varios pacientes más de una manifestación clínica.

En 7 de los 37 enfermos se hizo además el diagnóstico de depresión moderada a severa, con

\section{Tabla 1. M anifestaciones clínicas en 37 pacientes con enfermedad celíaca}

\begin{tabular}{|lrr|}
\hline & $\mathrm{n}$ & $\%$ \\
\hline Diarrea & 29 & 78 \\
Dolor abdominal & 14 & 38 \\
Baja de peso & 14 & 38 \\
Distensión abdominal & 10 & 27 \\
Esteatorrea & 8 & 21 \\
Fatigabilidad & 6 & 16 \\
Edema & 3 & 8 \\
\hline
\end{tabular}


Tabla 2. Resultados de laboratorio en 37 pacientes con enfermedad celíaca

\begin{tabular}{|lcc|}
\hline & $\mathrm{x}$ (rango) & $\%$ alterado \\
\hline Hematocrito (\%) & $36,4(19,9-49,1)$ & $49 \%$ (hcto. $<37)$ \\
VHS (mm/h) & $24,1(1-98)$ & $57 \%(\mathrm{VHS}>10)$ \\
Albúmina (g/dl) & $3,2(0,7-4,6)$ & $46 \%(\mathrm{alb} .<3,5)$ \\
Transaminasa oxaloacética (U/L) & $48,6(9-334)$ & $38 \%(>38 \mathrm{U} / \mathrm{L})$ \\
Transaminasa pirúvica (U/L) & $47,9(1-218)$ & $27 \%(>41 \mathrm{U} / \mathrm{L})$ \\
Fosfatasas alcalinas (U/L) & $182,5(55-511)$ & $54 \%(>129 \mathrm{U} / \mathrm{L})$ \\
\hline
\end{tabular}

control y tratamiento psiquiátrico. Además, se diagnosticó hipotiroidismo en cinco casos, síndrome de Sjögren en tres, osteoporosis en tres, diabetes tipo 1 y cirrosis biliar primaria en dos casos y en uno hepatitis crónica. En 19 pacientes, en quienes la diarrea era el síntoma principal, se realizó una colonoscopia con biopsias, encontrando 4 casos de colitis microscópica y un caso de colitis ulcerosa.

Los principales hallazgos de laboratorio se presentan en la Tabla 2. Destaca la presencia de anemia, definida por hematocrito $<37 \%$ en 18 $(49 \%)$ casos, velocidad de sedimentación $>10$ $\mathrm{mm} / \mathrm{h}$ en 21 (57\%), hipoalbuminemia $<3,5 \mathrm{~g} / \mathrm{dl}$ en 17 (46\%), elevación de fosfatasas alcalinas en 20 (54\%), transaminasa oxaloacética en 14 (38\%) y transaminasa pirúvica en $10(27 \%)$ casos.

El esteatocrito se midió en 17 pacientes, siendo positivo en 13 (76\%) de ellos.

La determinación de anticuerpos mostró positividad para los anticuerpos $\mathrm{AE}$ en 17 de 22 pacientes (77\%) y para los anticuerpos ATG en 19 de 22 pacientes (86\%). Al sumar el resultado de anticuerpos AE y ATG éstos resultaron positivos en 29 de 32 pacientes (90\%).

La endoscopia fue sugerente de EC en 16 de 34 pacientes (47\%), básicamente por hallazgo de disminución subjetiva de los pliegues de Kerckring en 13 pacientes y en 6 de ellos por la presencia de un patrón nodular de la mucosa 0 aspecto de empedrado.

La biopsia duodenal confirmó el diagnóstico por la presencia de atrofia vellositaria de distintos grados en 34 (92\%) de los 37 pacientes: Marsh IIIA en $13(35,1 \%)$, IIIB en $18(48,6 \%)$ y Marsh IIIC en $3(8,1 \%)$. Encontramos tres casos $(8,1 \%)$ de pacientes con histología normal. El primer caso corresponde a un hombre que presentaba diarrea crónica con baja de peso (IMC 19) y anticuerpos

\section{Tabla 3. C aracterísticas histológicas en} 37 pacientes con enfermedad celíaca

\begin{tabular}{|lrr|}
\hline & $\mathrm{n}$ & $\%$ \\
\hline Histología normal & 3 & 8,1 \\
Marsh I & 0 & \\
Marsh II & 0 & \\
Marsh III A & 13 & 35,1 \\
Marsh III B & 18 & 48,6 \\
Marsh III C & 3 & 8,2 \\
\hline
\end{tabular}

Marsh (ver referencia 3).

ATG positivos. El segundo paciente, también hombre, consultó por diarrea y meteorismo y presentaba serología AE y ATG positivas. El tercer caso corresponde a una mujer con historia de diarrea de 5 años de evolución y anticuerpos ATG positivos. Los tres pacientes tuvieron respuesta clínica favorable al régimen sin gluten, con desaparición de la diarrea y mejoría del estado nutricional en el primer caso (Tabla 3).

\section{Discusión}

Con la introducción de los métodos serológicos para el diagnóstico de EC, se ha observado que la prevalencia de ésta en países europeos y algunos latinoamericanos (Argentina), es mucho mayor que la publicada previamente y corresponde a 1 en 99 a 300 personas $^{4}$. En un estudio en niños, en Italia, mediante el examen de anticuerpos antigliadina y antiendomisio, se encontró una prevalencia de 1 en 184, con una relación de 1 caso conocido 
por cada 7 desconocidos. Maki et al encontraron en Finlandia una prevalencia estimada de la enfermedad de 1 en 99 estudiantes entre 7 y 16 años, mediante screening con anticuerpos AE y ATG y posterior confirmación histológica ${ }^{5-7}$.

En Chile no existen estudios de prevalencia en población general y el aumento de casos en el último tiempo sugiere un elevado número de pacientes subdiagnosticados.

En una revisión reciente en pacientes adultos en Estados Unidos, la edad promedio de diagnóstico en un grupo de 1.032 pacientes fue de 46 años, siendo los síntomas más prevalentes fatigabilidad, dolor y distensión abdominal. El 37\% de estos pacientes había sido diagnosticado previamente como portadores de Síndrome de Intestino Irritable $^{8}$. Otro estudio reveló que el porcentaje de diarrea como manifestación de la enfermedad fue significativamente menor en aquellos pacientes diagnosticados por serología después del año 1993, lo que sugiere un diagnóstico más precoz ${ }^{9}$.

En nuestra serie, el porcentaje de pacientes con diarrea fue de $78 \%$, con $21 \%$ de pacientes que refirió esteatorrea clínica. Sin embargo, en $76 \%$ de los pacientes en quienes se midió el esteatocrito el resultado fue positivo. Se debe destacar también que se encontraron 4 casos de colitis microscópica y un caso de colitis ulcerosa asociados, lo que sugiere la importancia de solicitar serología para $\mathrm{EC}$ en estos casos. Por otro lado, también un porcentaje elevado de pacientes se presentó sin diarrea y con síntomas inespecíficos como dolor abdominal difuso, distensión abdominal y fatigabilidad. Por lo tanto, es probable que un número de pacientes se manifieste como trastorno funcional y no sea mayormente investigado. Además, debe considerarse que un porcentaje importante de estos pacientes cursa con trastornos del ánimo. En nuestra serie llama la atención la presencia de siete casos (19\%) de depresión entre moderada y severa con diagnóstico psiquiátrico y en tratamiento farmacológico, lo que puede confundir aún más el cuadro clínico. Así mismo, es destacable la presencia de otras patologías inmunológicas como hepatopatías crónicas e hipotiroidismo.

El porcentaje de pacientes con bajo peso (29\%) fue similar a otras series, así como la edad promedio de diagnóstico (41 años) ${ }^{8}$.

Respecto a los hallazgos de laboratorio, el número de pacientes con algún grado de anemia
(49\%) concuerda con lo publicado en la literatura, que sugiere que la anemia por deficiencia de fierro es una manifestación frecuente en adultos. Karnam encontró $2,8 \%$ de pacientes celíacos no sospechados en un estudio prospectivo en adultos con anemia ferropriva en quienes se realizó endoscopia con biopsias duodenales y anticuerpos $\mathrm{AE}^{10}$.

La alteración de las pruebas hepáticas se considera también una manifestación frecuente, siendo lo más relevante la elevación de las fosfatasas alcalinas y de las transaminasas, que probablemente refleja grados variables de esteatosis o esteatohepatitis. En nuestros pacientes, encontramos aumento de fosfatasas alcalinas en más de la mitad de los enfermos y de la transaminasa oxaloacética en 38\%. De hecho dos pacientes asintomáticos desde el punto de vista intestinal, se diagnosticaron en el estudio por alteración de las pruebas hepáticas ${ }^{2}$.

En el presente estudio, encontramos un buen rendimiento diagnóstico de los anticuerpos $\mathrm{AE}$ y ATG con 77 y $86 \%$ de positividad respectivamente, medidos en 22 de los 37 pacientes, que aumentó a un $90 \%$ cuando se realizaron ambos exámenes. Estos hallazgos concuerdan con un estudio en 49 pacientes celíacos que mostró sensibilidad 89 y 92\% para los AE y ATG respectivamente y especificidad de $100 \%$ para ambos exámenes ${ }^{11}$.

Se ha visto que la inspección cuidadosa del duodeno en la endoscopia permitiría sospechar EC. En una serie de 88 pacientes con diagnóstico histológico confirmado se encontró en 79,5\% de pacientes, examinados en forma dirigida en la endoscopia, un aspecto macroscópico sugerente de la enfermedad. Más aún, en 13 de ellos (15\%) el diagnóstico de EC se hizo sólo por el hallazgo endoscópico. Por lo tanto, aun cuando la sensibilidad de la endoscopia como test de screening podría considerarse baja, permite plantear el diagnóstico en un grupo de pacientes derivados por otras causas, agregando biopsias de duodeno y serología en los casos sospechosos. Las características endoscópicas principales son la disminución de los pliegues de Kerckring, el patrón nodular de la mucosa o aspecto de empedrado. En nuestra serie, 47\% de los pacientes presentaron el aspecto endoscópico sugerente de EC, en su mayoría por disminución de los pliegues ${ }^{12}$.

Respecto a los hallazgos histológicos, la biopsia se considera diagnóstica al mostrar 
atrofia vellositaria de distintos grados en un contexto clínico y serológico adecuado. Sin embargo, un estudio reciente sugiere que en pacientes de alto riesgo, tanto adultos como niños y con cuadro clínico muy sugerente de EC y serología positiva, las alteraciones de la mucosa pueden no estar presentes para confirmar el diagnóstico. Los tres pacientes con clínica sugerente de EC y serología positiva, en quienes la biopsia mostró una mucosa duodenal normal podrían encontrarse en la fase latente de la enfermedad ${ }^{13}$.

El espectro histológico en la EC abarca desde la histología normal pasando por el aumento de linfocitos intraepiteliales hasta la atrofia vellosita-

\section{REFERENCIAS}

1. TRIER J. Celiac Sprue and Refractory Sprue. En Sleisenger y Fordtran's ed. Gastrointestinal and Liver Disease. Editorial W.B. Saunders Company, 1998; 1557-73.

2. Farrel R, Ciaràn K. Celiac Sprue. N Engl J Med 2002; 346: 180-8.

3. Oberhuber G, Granditsch G, Vogelsang H. The histopathology of celiac disease: time for a standardized report scheme for pathologists. Eur J Gastroenterol Hepatol 1999; 11: 1185-94.

4. Fasano A, Catassi C. Current approaches to diagnosis and treatment of celiac disease: an evolving spectrum. Gastroenterology 2001; 120: 636-51.

5. Catassi C, Fabiani E, Ratsch IM, Coppa GV, Giorgi PL, Pierdomenico R et al. The coeliac iceberg in Italy. A multicentre antigliadin antibodies screening for celiac disease in schoolage subjects. Acta Paediatr Suppl 1996; 412: 29-35.

6. Maki M, Mustalahati $\mathrm{K}$, KokKonen J, Kulmala $\mathrm{P}$, Haapalahti M, Karttunen T et al. Prevalence of celiac disease among children in Finland. NEngl J Med 2003; 348: 2517-24.

7. Fasano A. Celiac disease. How to handle a clinical chameleon? N Engl J Med 2003; 348: 2568-70. ria parcial o total. Por otra parte, las lesiones mucosas pueden tener distribución heterogénea por lo que una biopsia negativa no descarta el diagnóstico en presencia de un cuadro clínico y de laboratorio compatible ${ }^{14}$.

En conclusión, en el grupo de pacientes celíacos adultos los síntomas pueden apartarse de lo clásico, siendo destacable la presencia de anemia ferropriva y la alteración de las pruebas hepáticas. En presencia de estas alteraciones la EC debe ser considerada en el diagnóstico diferencial.

Por último el uso de exámenes serológicos puede ayudar a identificar nuevos pacientes, dado su alto rendimiento diagnóstico.

8. Zipser RD, Patel S, Yahya KZ, Baisch DW, Monarca E. Presentations of adult celiac disease in a nationwide patient support group. Dig Dis Sci 2003; 48: 761-4.

9. Lo W, Sano K, Lebwohl B, Diamond B, Green PH. Changing presentation of adult celiac disease. Dig Dis Sci 2003; 48: 395-8.

10. Karnam US, Fólder LR, Raskin JB. Prevalence of occult celiac disease in patients with iron-deficiency anemia: a prospective study. South Med J 2004; 97: 30-4.

11. Weitz JC, Montalva R, Alarcón T, Contreras L. Determinación de anticuerpos anti-transglutaminasa en el diagnóstico de enfermedad celíaca. Rev Méd Chile 2003; 131: 31-6.

12. Vjero K, Martucci S, Alvisi C, Broglia F, Torelo F, PeREgo M ET al. Defining a proper setting for endoscopy in coeliac disease. Eur J Gastroenterol Hepatol 2003; 15: 675-8.

13. Scoglio R, Di Pasquale G, Pagano G, Lucanto MC, Magazzù G, SFeriazzas C. Is intestinal biopsy always needed for diagnosis of celiac disease? Am J Gastroenterol 2003; 98: 1325-31.

14. Kakar S, Nehra V, MurRia JA, Dayharsh GA, BURGaRT LJ. Significance of intraepithelial lymphocytosis in small bowel biopsy samples with normal mucosal architecture. Am J Gastroenterol 2003; 98: 2027-33. 